\title{
Electron cloud instability in high intensity proton rings
}

\author{
K. Ohmi, T. Toyama, and C. Ohmori \\ KEK, 1-1 Oho, Tsukuba, 305-0801, Japan \\ (Received 3 July 2002; published 20 November 2002)
}

\begin{abstract}
An $e^{-} p$ instability has been observed in some proton rings. The instability, which causes beam loss, limits the performance of the ring. The instability may be serious for 3 and $50 \mathrm{GeV}$ proton storage rings in the Japan Proton Accelerator Research Complex (J-PARC). We study the $e^{-} p$ instability in several high intensity proton storage rings operated in the world. This work informs J-PARC of the necessity to cure the instability, for example, by applying a TiN coating on the chamber surface.
\end{abstract}

DOI: 10.1103/PhysRevSTAB.5.114402

PACS numbers: 29.27.Bd, 29.20.Lq

\section{INTRODUCTION}

The electron-proton $\left(e^{-} p\right)$ instability has been discussed for a long time. The first work was done at CERN-ISR [1,2]. An instability was observed in the coasting beam operation and it was cured using clearing electrodes. After that, an instability was observed for bunched proton beam in a proton synchrotron ring at Los Alamos National Laboratory (LANL-PSR) [3]. They reported that the instability was caused by an electron cloud. An instability, which seems to be due to $e^{-} p$, has also been observed at the AGS booster [4]. In their scenario, primary electrons were produced by proton losses at the chamber surface, and an electron cloud was formed by the trailing-edge multipacting $[3,5,6]$. Electrons, which are produced by the head part of the beam, are accelerated by the body part of the beam, and released at the trailing edge. The accelerated electrons create secondary electrons at the chamber surface. The secondary electrons are amplified at every hitting of the chamber wall.

A high intensity proton accelerator facility has been proposed in Japan as a joint project of KEK and JAERI. The facility, which is named the Japan Proton Accelerator Research Complex (J-PARC), is equipped with two proton rings: a $3 \mathrm{GeV}$ rapid cycle synchrotron and a $50 \mathrm{GeV}$ proton synchrotron. The bunch population, which is $4.15 \times 10^{13}$, is comparable with that of PSR. The electron cloud instability may be serious for these two rings of J-PARC. We discuss the electron cloud effects for J-PARC mainly in this paper.

In the U.S., the Spallation Neutron Source (SNS), which is equipped with a high intensity proton synchrotron, is under construction at ORNL. Many studies on the electron cloud effects have been done for SNS [7-9].

The electron cloud instability has not been observed in all high intensity proton rings. For example, the instability has not been observed at the rapid cycle synchrotron ISIS in Rutherford Laboratory at bunch intensity comparable with PSR. AGS has an intensity which is only slightly different from that of J-PARC $50 \mathrm{GeV}$, but the instability has not been observed. It is worthwhile to compare these proton rings from the point of view of the electron cloud instability. The parameters of these rings are summarized in Table I.

The electron cloud causes both coupled and single bunch instabilities. A perturbation of the cloud induced by a bunch affects other bunches and causes the coupled bunch instability. A perturbation induced by a part of a

TABLE I. Basic parameters of the proton rings.

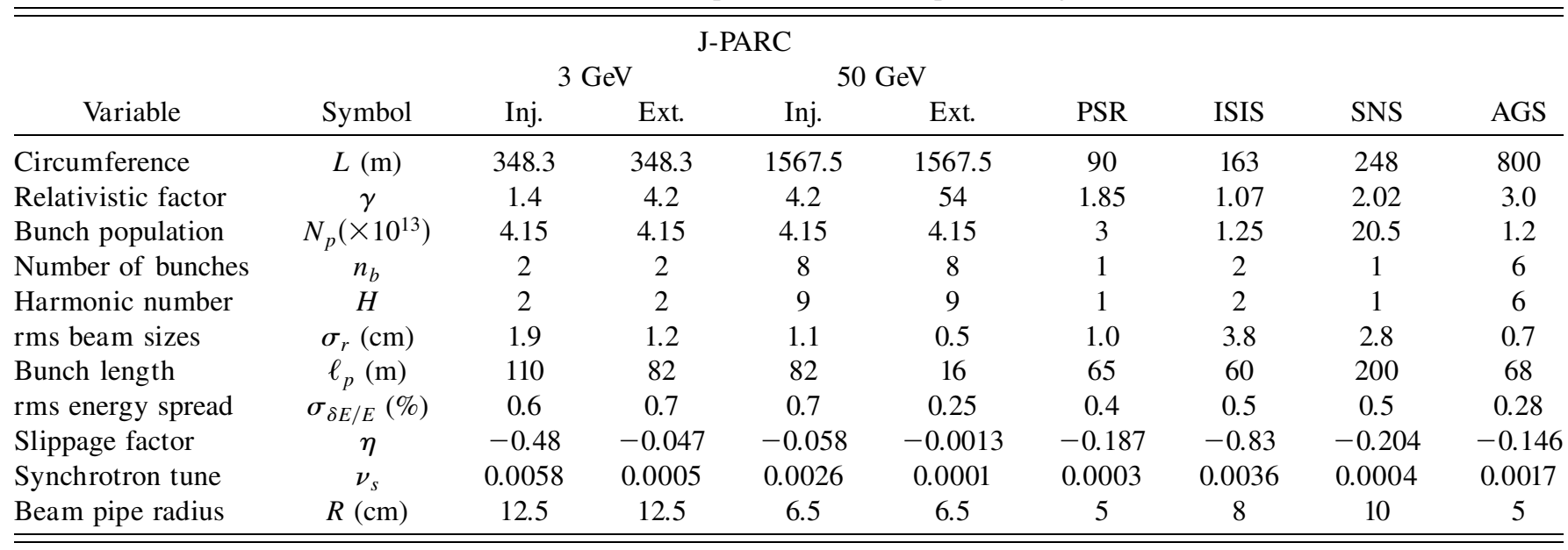


bunch affects another part of the same bunch and causes the single bunch instability. In these rings, the bunch length and the free space between bunches are both several tens of meters. At first sight, the free space of several $10 \mathrm{~m}$ seems to be long enough to smear out perturbation of the bunch. Hence, we focus on the single bunch instability in this paper.

We discuss formation of the electron cloud in Sec. II. Electrons created by ionization and proton loss are taken into account as the primary ones. Secondary electrons are created by the original electrons hitting the chamber wall. The electron cloud density of each ring is estimated by a computer simulation considering the primary and secondary electrons. Our simulations are performed in a field-free region mainly, and the contribution of the magnetic field is also investigated. We study the instability of the proton beam interacting with the electron cloud in Sec. III. We analyze the instability with a tracking simulation [10] and a wake field approach [11].

\section{FORMATION OF ELECTRON CLOUD}

We discuss electron production and formation of the cloud. Many possibilities for primary electron production are considered. Ionization of residual gas due to the proton beam creates electrons and ions. The ions create electrons when they are absorbed at the chamber surface $[12,13]$. Electrons are also created by proton absorption at the beam chamber surface. $\mathrm{H}^{-}$injection is a direct electron source.

The yield of ionization electrons is determined by the ionization cross section and the vacuum pressure in the beam chamber. Electrons are produced along the beam trajectory. Electron production at the chamber surface is rather complex. It is not well known how many electrons are produced by a proton beam, though there are many candidates. Macek et al. measured the number of electrons hitting the chamber wall using button electrodes at PSR [3]. They observed a peak current of $400 \mu \mathrm{A} / \mathrm{cm}^{2}$ with a width of $50 \mathrm{~ns}$ at a revolution period of $350 \mathrm{~ns}$ with a proton current of $20 \mathrm{~A}$. In the positron machine, KEKBLER, we observed an electron current of $1-10 \mu \mathrm{A} / \mathrm{cm}^{2}$ (dc) at the positron current of $600 \mathrm{~mA}$. These measurements show that the number of electrons produced in high intensity proton rings is comparable to that of positron storage rings. In our first impression, it is surprising that proton rings have such a highly efficient electron production mechanism.

In KEKB, the electron current is understood to be due primarily to photoelectron emission caused by synchrotron radiation. The probability for a positron to emit a synchrotron radiation photon over a travel distance of $1 \mathrm{~m}$ is 0.15 , and $10 \%$ of the photons create photoelectrons: i.e., the electron production rate is $Y_{1}=1.5 \times$ $10^{-2} e^{-} /\left(\mathrm{m} \cdot e^{+}\right)$. The observed current value agrees well with simulations taking into account the electron yield, their motion, and the geometry of the button electrodes.

To explain the observed current in a proton machine, Macek et al. proposed electron production due to proton loss at the chamber surface [3]. Furman et al. [6] use an electron production rate of $Y_{1}=4.4 \times 10^{-6} e^{-} /(\mathrm{m} \cdot p)$ on the chamber surface for a proton traveling at a meter. They have observed proton loss of $4 \times 10^{-6}$ per turn at PSR $(L=90 \mathrm{~m})$. They assume that a proton creates 100 electrons per loss. Though this rate is still smaller than that of KEKB, multipacting due to secondary electrons makes up the difference. The number of amplified electrons was consistent with the electron current measurement.

Since we do not have clear information about the rate for J-PARC, we use this primary electron yield, $Y_{1}=$ $4.4 \times 10^{-6} e^{-} /(\mathrm{m} \cdot p)$, in our calculation, although this value may depend on energy, chamber geometry, surface condition, etc.

We also investigate ionization electrons. These electrons are produced by the ionization of residual gas in the chamber. Ionization cross section for $\mathrm{CO}$ and $\mathrm{H}_{2}$ is estimated to be $\sigma(\mathrm{CO})=1.3 \times 10^{-22} \mathrm{~m}^{2}$ and $\sigma\left(\mathrm{H}_{2}\right)=$ $0.3 \times 10^{-22} \mathrm{~m}^{2}$ using the Bethe formula [14]. The molecular density $d_{m}$ is related to the partial pressure in $\mathrm{nPa}$ using the relation at $20^{\circ} \mathrm{C}, d_{m}\left(\mathrm{~m}^{-3}\right)=$ $2.4 \times 10^{11} P_{m}(\mathrm{nPa})$. The electron production rate is $7.7 \times$ $10^{-9} e^{-} /(\mathrm{m} \cdot p)$ at $2 \times 10^{-7} \mathrm{~Pa}$. The production rate is 7 orders of magnitude smaller than that of the photoelectron in KEKB and is 3 orders smaller than that of the proton loss in PSR.

Secondary electron production plays an important role for electron cloud buildup in proton rings, because primary electrons are much less common than in positron rings. Secondary yield, $\delta_{2}(E)$, which is the number of electrons created by an electron incidence with an energy $(E)$, is approximated by the formula [15],

$$
\delta_{2}(E)=\delta_{2, \max } \frac{E}{E_{\max }} \frac{1.44}{0.44+\left(E / E_{\max }\right)^{1.44}} .
$$

Figure 1 shows the secondary yield for $\delta_{2, \max }=2.1$ and $E_{\max }=200 \mathrm{eV}$. These values are obtained for aluminum by measurements [16].

In recent studies, an elastic reflection of electrons is taken into account by $\delta_{2}(0)=0.5 \sim 0.8$ [17]. In this paper, we use the original formula, Eq. (1), therefore $\delta_{2}(0)=0$.

\section{Simulation of electron cloud formation}

Electron cloud formation is estimated by tracking the motion of electrons produced by the primary and the secondary electron emissions $[15,18]$. The motion of electrons is calculated in the transverse plane. We consider an electron cloud distribution at the position $s_{e}$. The distribution and line density $\lambda_{e}(z)$ is assumed to depend 


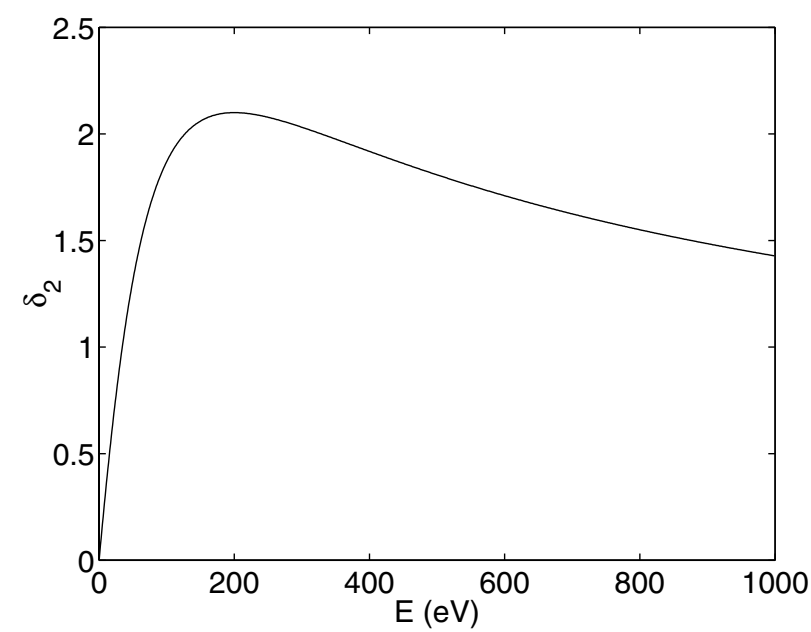

FIG. 1. Secondary electron yield depending on incident electron energy for $\delta_{2, \max }=2.1$ and $E_{\max }=200 \mathrm{eV}$.

only on $z=s_{e}-v t$, but to be independent on $s_{e}$ for the choice of independent variables $(z, s)$, where $v$ is the velocity of protons. We neglect the space charge force between electrons in the present simulation, because the average neutralization factor $\lambda_{e} / \bar{\lambda}_{p}$ is of the order of 0.1 in our case as seen later, where $\lambda_{e}$ and $\bar{\lambda}_{p}$ are the electron line density and the proton average line density, respectively. Therefore the results shown below scale linearly with the yield of primary electrons. However electron motion during the absence of the beam may be important for the surviving electrons, which are the seeds for the electron cloud generated by the next bunch. We will include the space charge force in the near future. The magnetic field may affect the electron cloud formation. We first treat the electron cloud buildup for the field-free region and then discuss the effects of the magnetic field.
The proton beam is sliced along the longitudinal direction into equal-size steps. Each slice has a local proton density $\lambda\left(z_{i}\right)_{p}$, where $\lambda\left(z_{i}\right)_{p}$ is a periodic function of the bunch period and is $\lambda\left(z_{i}\right)_{p}>0$ for $0<z<\ell_{p}$. Electrons are tracked step by step along the passage of the proton beam. The equation of motion for electrons is expressed by

$$
\frac{d^{2} \boldsymbol{x}(t)}{d t^{2}}=-2 \lambda_{p}\left(s_{e}-v t\right) r_{e} c^{2} \boldsymbol{F}_{G}[\boldsymbol{x}(t)],
$$

where $r_{e}$ is the classical electron radius. The force $\boldsymbol{F}_{G}(\boldsymbol{x})$ is expressed by the Bassetti-Erskine formula normalized so that $\boldsymbol{F}_{G} \rightarrow \boldsymbol{x} /|\boldsymbol{x}|^{2}$ as $\boldsymbol{x} \rightarrow \infty$. $t$ is the time when the beam slice at $z=s_{e}-v t$ arrives at the electron position $s_{e}$. The equation is integrated with the time step of the slice width. We use 1000 slices for the proton beam in this paper.

The electrons are produced at the chamber surface or at the beam position, when the proton beam passes through the longitudinal positions. The number of productions is proportional to the local proton density. We produce primary macroelectrons of $1.0-1.5 \times 10^{4}$ for a proton bunch passage in this simulation. The longitudinal charge distribution of the proton bunch is expressed by a sinusoidal function in our simulation as

$$
\rho_{p}=\frac{\pi N_{p}}{2 \ell_{p}} \sin \frac{\pi z}{\ell_{p}} .
$$

The number of electrons in the chamber is calculated during the passage of ten proton bunches.

We first discuss the cloud formation for the electron production at the chamber surface. Figure 2 shows the number of electrons as a function of $s$. The vertical axis is the number of electrons which is normalized by the
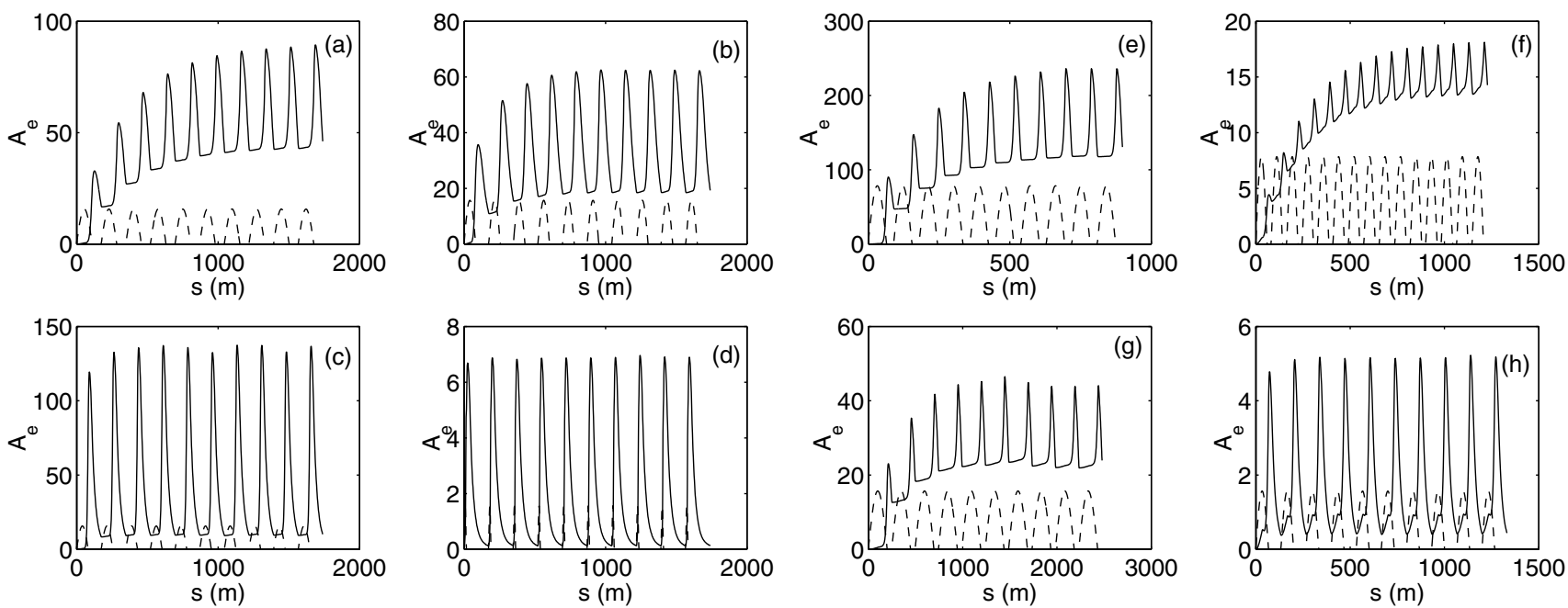

FIG. 2. Electron amplification factor. The proton beam profiles are plotted by the dashed lines in the pictures with arbitrary units. Electrons are produced at the chamber surface. (a) $3 \mathrm{GeV}$ injection; (b) $3 \mathrm{GeV}$ extraction; (c) $50 \mathrm{GeV}$ injection; (d) $50 \mathrm{GeV}$ extraction; (e) PSR; (f) ISIS; (g) SNS; (h) AGS. 

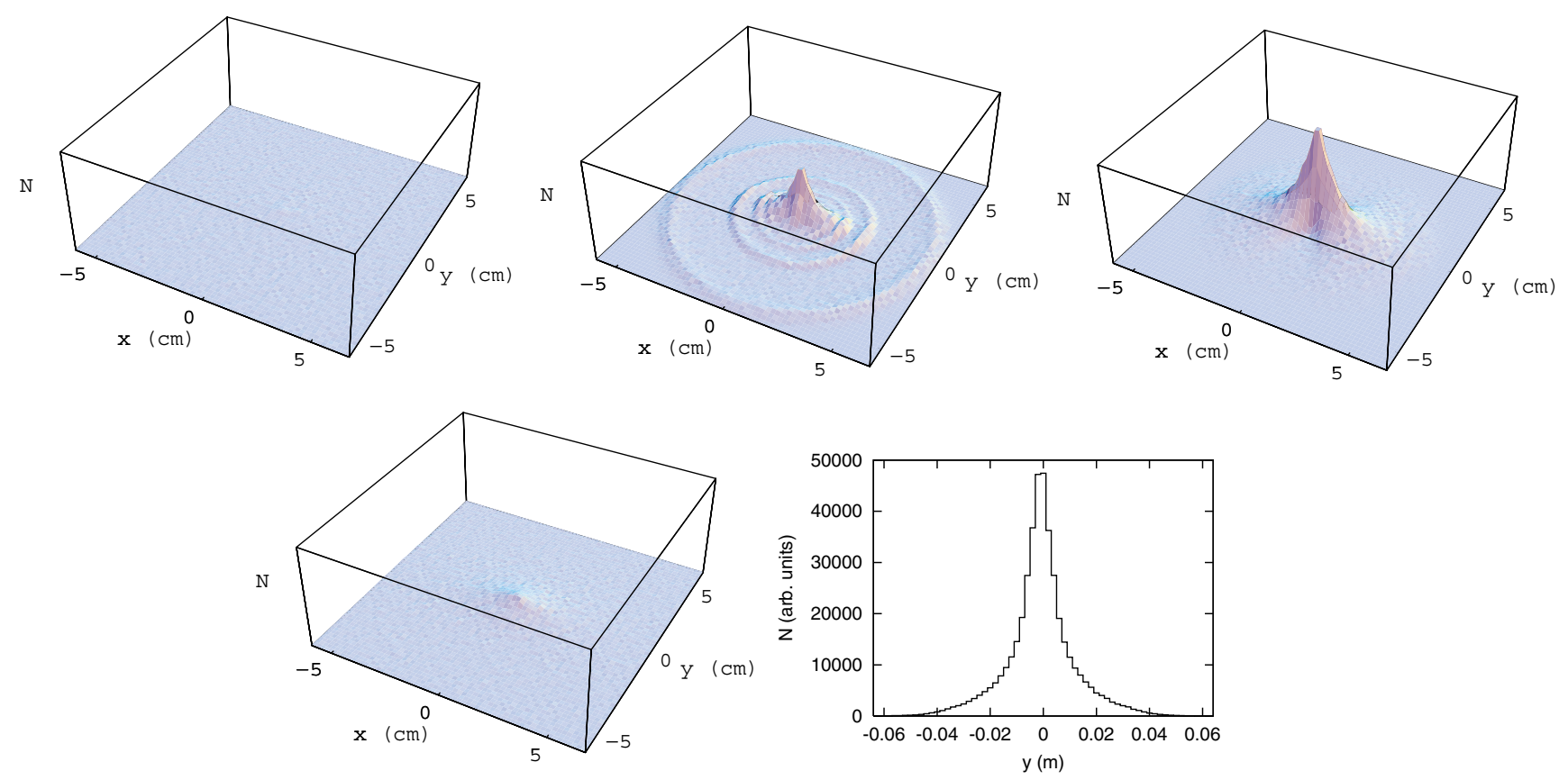

FIG. 3. (Color) Transverse electron distribution during the passage of the last (10th) bunch: (1st) $0 \mathrm{~m}$; (2nd) $10 \mathrm{~m}$; (3rd) 50 m; (4th) $100 \mathrm{~m}$; and (5th) vertical distribution after $50 \mathrm{~m}$ passage. The electron density, $N$, is plotted with arbitrary units in the $1 \mathrm{st}$ to 4 th pictures.

number of primary electrons produced by a bunch, $A_{e}(z)=\lambda_{e}(z) /\left(Y_{1} N_{p}\right) . A_{e}$ characterizes the amplification factor due to secondary emission.

The electron cloud density gradually increases at the beginning of the interaction and suddenly increases at the bunch tail. This is due to the trailing-edge multipacting [6]. The number of electrons decreases after the end of the multipacting, but a considerable quantity of electrons remains in the chamber at the arrival of the next bunch. This quantity depends on the ring and beam parameters. Electrons accumulate gradually again with a sudden leap at the tail of the 2 nd bunch. These processes, in which the number of electrons increases and decreases, are repeated at every passage of the bunches. The numbers of electrons at the top and bottom reach an equilibrium value after the passage of 5-10 bunches. The equilibrium value and the number of bunch passages required to reach it depend on the ring and beam parameters.

Figure 3 shows the transverse distribution of electrons during the passage of the last (10th) bunch for J-PARC $50 \mathrm{GeV}$ at injection. The figure shows that electrons are distributed widely at the start of the interaction with the bunch and are gathered at the beam position immediately and splash after the interaction. The last picture shows the vertical distribution of the electrons after $50 \mathrm{~m}$ passage. The cloud size is comparable to the beam size.

We also investigated electron cloud buildup in the case that initial electrons were produced at the beam position. Electrons produced by the ionization correspond to this case. The initial energy of the ionization electron is neglected. Figure 4 shows the amplification of electrons, $A_{e}$. The number is far less than that produced at the chamber. This means that electrons cannot get sufficient energy to produce secondary electrons. The number should be increased when the reflection of electrons is taken into account. The contribution of electrons produced at the beam position can be regarded as the correction for the production efficiency at the chamber wall, since the secondary electrons are produced at the chamber wall.
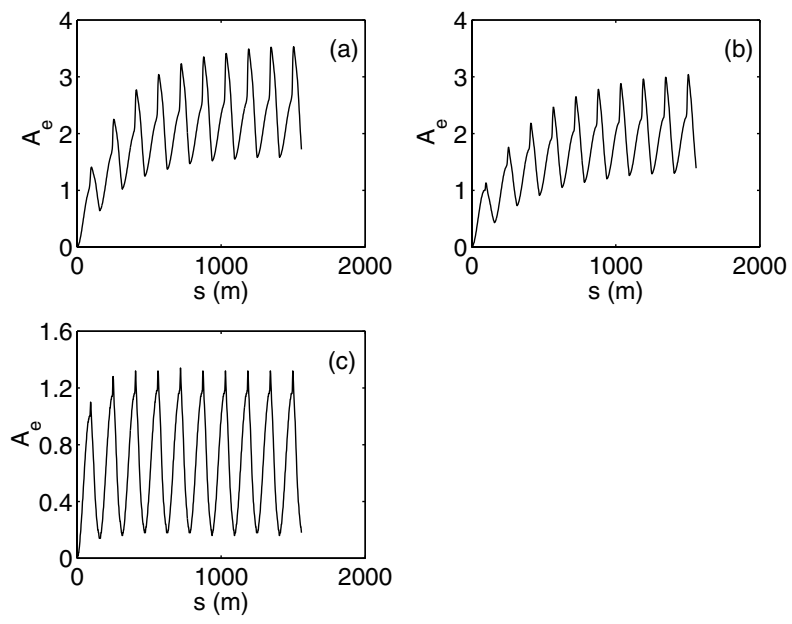

FIG. 4. Electron amplification factor. Electrons are produced at the beam position. (a) $3 \mathrm{GeV}$ injection; (b) $3 \mathrm{GeV}$ extraction; (c) $50 \mathrm{GeV}$ injection. 
TABLE II. Electron cloud buildup of the proton rings.

\begin{tabular}{|c|c|c|c|c|c|c|c|c|}
\hline \multirow[b]{3}{*}{ Variable } & \multicolumn{4}{|c|}{ J-PARC } & \multirow[b]{3}{*}{ PSR } & \multirow[b]{3}{*}{ ISIS } & \multirow[b]{3}{*}{ SNS } & \multirow[b]{3}{*}{ AGS } \\
\hline & \multicolumn{2}{|c|}{$3 \mathrm{GeV}$} & \multicolumn{2}{|c|}{$50 \mathrm{GeV}$} & & & & \\
\hline & Inj. & Ext. & Inj. & Ext. & & & & \\
\hline$A_{e}$ (bottom) & 42.0 & 18.0 & 9.4 & 0.13 & 118.0 & 12.9 & 21.9 & 0.42 \\
\hline$A_{e}(\mathrm{peak})$ & 87.6 & 62.0 & 136.0 & 6.9 & 236.0 & 17.5 & 44.1 & 5.18 \\
\hline$f$ (bottom) & 0.020 & 0.0067 & 0.0035 & 0.00001 & 0.034 & 0.003 & 0.019 & 0.0001 \\
\hline$f$ (peak) & 0.042 & 0.023 & 0.05 & 0.0005 & 0.067 & 0.005 & 0.039 & 0.0015 \\
\hline
\end{tabular}

We now estimate the neutralization factor of the electron cloud for a proton beam. The proton and electron line densities are functions of $z$. The neutralization factor is defined as the electron cloud line density divided by the average proton line density, $f(z)=\lambda_{e}(z) / \bar{\lambda}_{p}$, which is a function of $z$. The neutralization factor is expressed by

$$
f(z)=\frac{A_{e}(z) Y_{1} N_{p}}{N_{p} / \ell_{p}}=A_{e}(z) Y_{1} \ell_{p} .
$$

Table II shows peak and bottom values of the neutralization factor for each ring. The neutralization factor strongly depends on the parameters: beam size, chamber size, bunch length, and bunch spacing. The results for PSR and SNS are qualitatively consistent with previous works $[6,7,9]$.

We discussed the electron cloud amplification for $\delta_{2, \max }=2.1$ so far. The secondary yield $\delta_{2}$ can be improved by surface treatments, for example, by applying a TiN coating. Similar calculations were done for various $\delta_{2, \max }$ to study how the secondary yield affects the electron cloud amplification. Figure 5 shows the electron amplification factor for $\delta_{2 \text { max }}=1.5,1.8$, and 2.1. We find out that the surface treatment to reduce $\delta_{2}$ is very efficient to cure the electron cloud effects.

We obtained some characteristics concerning the electron cloud in this simulation. They are summarized as follows: (i) The electron cloud reaches an equilibrium density after the passage of approximately ten bunches in most cases. (ii) The leading edge of the proton beam passes through the electron cloud which is formed by previous bunches. The electron cloud distributes all over
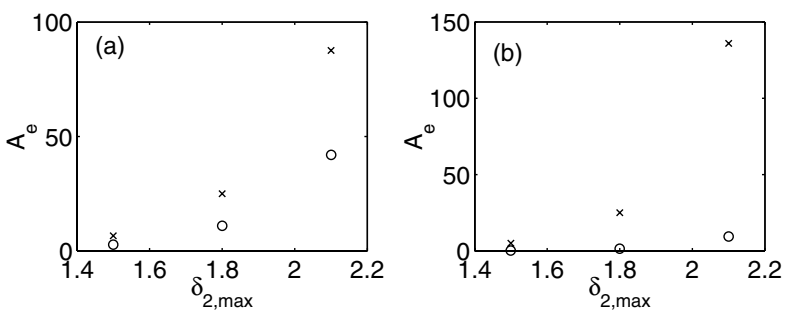

FIG. 5. Electron amplification factor for the secondary yield. The crosses and circles denote the peak and bottom values, respectively. (a) and (b) are for $3 \mathrm{GeV}$ injection and for $50 \mathrm{GeV}$ injection at J-PARC, respectively.
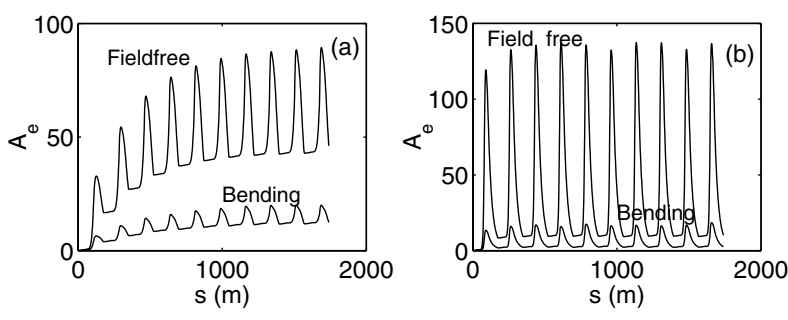

FIG. 6. Electron amplification factor in bending magnets. The amplification factors for the field-free regions are also plotted for the comparison. (a) and (b) are obtained for 3 and $50 \mathrm{GeV}$ injection at J-PARC, respectively.

the chamber. (iii) Electrons are gathered at the beam position immediately at the passage of the leading edge. (iv) The number of electrons suddenly increases by $2 \sim 10$ times depending on the beam and ring parameters at the trailing edge of the proton beam. (v) The electron amplification strongly depends on the secondary yield, $\delta_{2}$.

We now discuss the electron cloud buildup in magnetic field. The beam chamber is covered with various magnets as bending, quadrupole, and higher order magnets in actual rings. The field-free regions and bending magnets occupied the biggest part of the rings. In bending magnets with a strong dipole field, electrons undergo cyclotron motion with a small radius $(<1 \mathrm{~mm})$ and at a high frequency $(>10 \mathrm{GHz}$ ). Therefore we assume that electrons move along the vertical axes and repeat the same simulations. Figure 6 shows the amplification factor of electrons as a function of $s$ for 3 and $50 \mathrm{GeV}$ injection at J-PARC. In this calculation, dipole fields have the effect of suppressing the electron buildup. In our calculation, electrons are created uniformly at the chamber surface. A considerable fraction of the electrons cannot approach the beam in the model, therefore they do not get sufficient energies for the multipacting. The result may depend on the model. It may be possible that there is some special mechanism to trap [19] or to amplify electrons.

\section{INSTABILITY CAUSED BY ELECTRON CLOUD}

We discuss the instability caused by the electron cloud. We estimate the instability for the assumption that the 
whole of the ring is field free. The instability is studied by simulation using beam tracking and the wake field approach. We study the transverse dipole mode instability, in which the proton beam can have a dipole moment $\overline{\boldsymbol{x}}_{p}(z, s)$, where $\overline{\boldsymbol{x}}_{p}(z, s)$ is a function of $z$.

\section{A. Simulation using beam tracking}

The electron cloud is created and accumulated by the passage of several bunches as shown in the previous section. We study the motion of proton bunches interacting with the electron cloud using a tracking simulation. For simplicity, we use the characteristics of the electron cloud summarized in the previous section. Electrons are assumed to be always uniformly distributed with a certain density in the vacuum chamber at the beginning of their interaction with a proton bunch; that is, they do not have memory of their interactions with previous bunches. Similar tracking simulations have been done elsewhere [20-22].

A proton bunch is represented by macroparticles which are located along $z$ with equal spacing. Each macroparticle has a charge and a mass corresponding to the proton line density. The macroparticle can undergo dipole motion with a dipole moment characterized by $\overline{\boldsymbol{x}}_{p, i}\left(z_{i}, s\right)=$ $\left(\bar{x}_{p}, \bar{y}_{p}\right)$, but the emittance (size) is kept constant. The electron cloud is set at some position in the ring and is represented by a large number of pointlike macroparticles denoted by $\boldsymbol{x}_{e, a}\left(a=1, N_{e}\right)$. The electrons are initialized as a uniform distribution in the transverse plane.

The equation of motion is expressed by

$$
\begin{gathered}
\frac{d^{2} \overline{\boldsymbol{x}}_{p, i}}{d s^{2}}+K(s) \overline{\boldsymbol{x}}_{p, i}=-\frac{2 r_{p}}{\gamma} \sum_{a=1}^{N_{e}} \boldsymbol{F}_{G}\left(\overline{\boldsymbol{x}}_{p, i}-\boldsymbol{x}_{e, a} ; \boldsymbol{\sigma}\right) \delta\left(s-s_{e}\right), \\
\frac{d^{2} \boldsymbol{x}_{e, a}}{d t^{2}}=-2 r_{e} c \sum_{i=1}^{N_{s l}} \lambda_{p}\left(z_{i}\right) \Delta z \boldsymbol{F}_{G}\left(\boldsymbol{x}_{e, a}-\overline{\boldsymbol{x}}_{p, i} ; \boldsymbol{\sigma}\right) \delta\left[t-t\left(z_{i}, s_{e}\right)\right],
\end{gathered}
$$

where $r_{p}$ is the classical proton radius.

The motion of the macroelectrons and macroprotons is tracked during the beam passage. After that, macroprotons are transported by the lattice magnets and then interact with randomly initialized electrons again. This procedure is repeated in every interaction of the bunch with the cloud.

We performed the simulation for J-PARC 3 and $50 \mathrm{GeV}$ rings at injection. The neutralization factor was $2 \%$ and $4 \%$ for 3 and $50 \mathrm{GeV}$ rings, respectively. These values are the bottom values in Table II. Figure 7 shows the vertical dipole moment of a proton bunch $\bar{y}_{p}\left(z_{i}, s\right)$ along the longitudinal position $z$ at $s=20 \times L$. Excitations of a dipole mode with the frequency $\omega_{e}$ is seen. We obtained a similar signal for the horizontal moment.

Figure 8 shows the variation of the dipole moment, $J_{y}$, where $J_{y}$ is one-half of the Courant Snyder invariant, is the maximum value along $z$. The growth rate $T_{0} / \tau_{g}$ in the
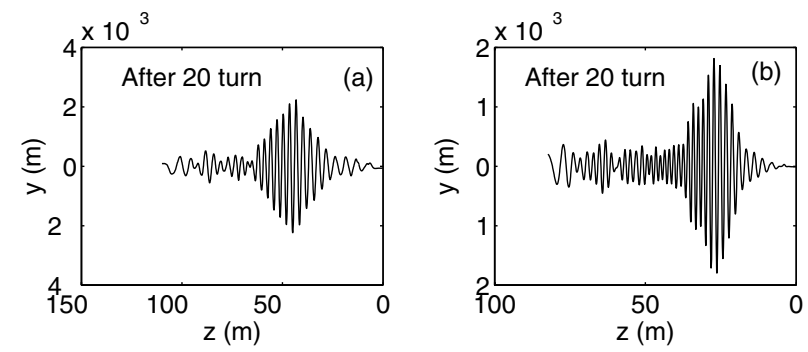

FIG. 7. Vertical dipole moments for J-PARC $3 \mathrm{GeV}$ and $50 \mathrm{GeV}$ rings at injection. The right-left correspond to the head-tail of a bunch, respectively. Pictures (a) and (b) are obtained for 3 and $50 \mathrm{GeV}$, respectively. revolution time, $T_{0}$, is $0.2\left(<0.1 \sigma_{r}\right) \sim 0.1\left(\approx 0.1 \sigma_{r}\right)$ for small amplitude in both cases.

To discuss the beam stability, we compare the growth rate with the Landau damping rate. We take into account the Landau damping caused by the longitudinal motion of a bunch, which disturbs the coherence of the dipole motion. The Landau damping rate is given by $\omega_{e} \eta \sigma_{\delta E / E}=\omega_{s} \omega_{e} \sigma_{z} / c$ for a long bunched beam [23]. The beam stability is estimated by these ratios, the growth rate divided by the Landau damping rate.

$$
U \equiv \frac{\sqrt{3}}{\Delta \omega \tau_{g}}=\frac{\sqrt{3} T_{0} / \tau_{g}}{2 \pi \nu_{s} \omega_{e} \sigma_{z} / c},
$$

where $\sqrt{3}$ is used as a normal stability condition.

For J-PARC 3 and $50 \mathrm{GeV}$ at injection, $U$ is obtained by the growth rate as

$$
U=0.15 \sim 0.07 \quad 3 \mathrm{GeV},
$$

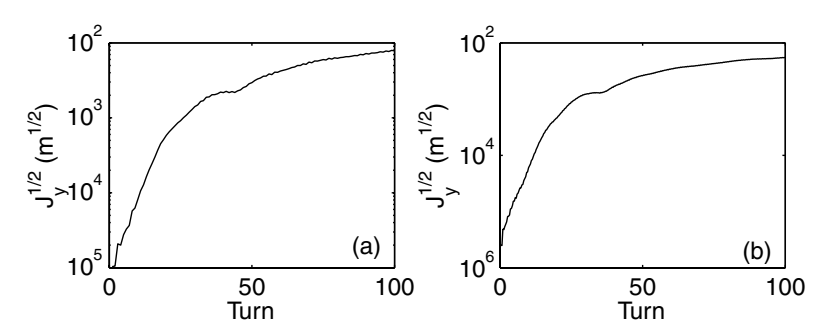

FIG. 8. Growth of the vertical dipole instability for J-PARC 3 and $50 \mathrm{GeV}$ rings at injection. The right-left correspond to the head-tail of a bunch, respectively. (a) and (b) are obtained for 3 and $50 \mathrm{GeV}$, respectively. 


$$
U=0.23 \sim 0.12 \quad 50 \mathrm{GeV} .
$$

$U$ 's are less than 1, therefore the proton beams interacting with the electron cloud are stable. Although the growths in Fig. 8 are very fast, Landau damping suppresses the instability because of large $\omega_{e} \sigma_{z} / c$.

\section{B. Wake field induced by electron cloud and beam stability}

Here we treat the instability with an analytic approach using the wake field induced by the electron cloud. We know that the electron cloud was gathered near the beam immediately at the beginning of the interaction with the beam, and the beam interacted with the pinched electron distribution during the passage through the cloud. The size of the electron cloud is about the same as the beam size. We regard the system as an interaction between the coasting beam and the electron cloud with transverse Gaussian distribution. We take the linear term of the interaction. Similar analysis has been done in previous works $[2,8,20,21,24,25]$.

The motions of the beam and the electron cloud are characterized by $y_{p}(s, z)$ and $y_{e}(s, t)$, respectively. The equations of motion for the beam and cloud are expressed as follows:

$$
\begin{gathered}
\frac{d^{2} y_{p}(s, z)}{d s^{2}}+\left(\frac{\omega_{\beta, y}}{c}\right)^{2} y_{p}(s, z)=-\left(\frac{\omega_{p, y}}{c}\right)^{2}\left\{y_{p}(s, z)-y_{e}[s,(s-z) / c]\right\}, \\
\frac{d^{2} y_{e}(s, t)}{d t^{2}}=-\omega_{e, y}^{2}\left[y_{e}(s, t)-y_{p}(s, s-c t)\right],
\end{gathered}
$$

where $\omega_{\beta, y}$ denotes the angular betatron frequency without electron interaction. The two coefficients $\omega_{p, y}$ and $\omega_{e, y}$ characterize the linearized force between the beam and the cloud, and are given by

$$
\omega_{p, y}^{2}=\frac{\lambda_{e} r_{p} c^{2}}{\gamma\left(\sigma_{x}+\sigma_{y}\right) \sigma_{y}}, \quad \omega_{e, y}^{2}=\frac{\lambda_{p} r_{e} c^{2}}{\left(\sigma_{x}+\sigma_{y}\right) \sigma_{y}},
$$

where $\lambda_{e}$ and $\lambda_{p}$ are the line densities of the cloud and the beam, and $\sigma_{x}$ and $\sigma_{y}$ are the horizontal and vertical beam sizes, respectively.

From Eqs. (10) and (11), an equation for the beam motion is obtained as follows:

$$
\frac{d^{2} y_{p}(s, z)}{d s^{2}}+\left(\frac{\tilde{\boldsymbol{\omega}}_{\beta}}{c}\right)^{2} y_{p}(s, z)=\frac{\omega_{p}^{2} \omega_{e}}{c^{3}} \int_{z}^{\infty} y_{p}\left(s, z^{\prime}\right) \sin \frac{\omega_{e}}{c}\left(z-z^{\prime}\right) d z^{\prime}
$$

Here $\tilde{\omega}_{\beta}^{2}=\omega_{\beta}^{2}+\omega_{p}^{2}$ is the angular betatron frequency including the frequency shift due to the electron cloud. The right-hand side of Eq. (13) can be represented by a wake function, which depends only on the longitudinal distance. Integrated over the ring circumference $L$, the wake function can be written as

$$
W_{1}(z)\left[\mathrm{m}^{-2}\right]=c R_{S} / Q \sin \left(\frac{\omega_{e}}{c} z\right),
$$

where

$$
c R_{S} / Q=\frac{\lambda_{e}}{\lambda_{p}} \frac{L}{\left(\sigma_{x}+\sigma_{y}\right) \sigma_{y}} \frac{\omega_{e}}{c} .
$$

This wake field does not damp for $z$ in this model, i.e., in the language of impedance, we would, say, that the $Q$ factor is infinite. Actually the frequency spread of $\omega_{e}$ should be taken into account. We add a damping term $2 \alpha y_{e}$ in the left-hand side of Eq. (11). The damping factor $\alpha$ corresponds to the frequency spread of $\omega_{e}$. The wake field is now expressed by

$$
W_{1}(z)=c \frac{R_{S}}{Q} \frac{\omega_{e}}{\tilde{\omega}} \exp \left(\frac{\alpha}{c} z\right) \sin \left(\frac{\tilde{\omega}}{c} z\right),
$$

where $\alpha=\omega_{e} / 2 Q$ and $\tilde{\omega}=\sqrt{\omega_{e}^{2}-\alpha^{2}}$. Note that $z<0$ for the backward direction.

In this framework the frequency spread ( $\alpha$ or $Q$ ) is not determined. The spread is caused by nonlinear interaction with the beam, the proton distribution along $z$, and the beam size modulation due to $\beta$ function variations. An estimation of $Q$ caused by nonlinear interactions is given in Ref. [11]. The wake field is calculated with the same simulation method. Figure 9 shows the wake field for J-PARC $50 \mathrm{GeV}$ at injection.

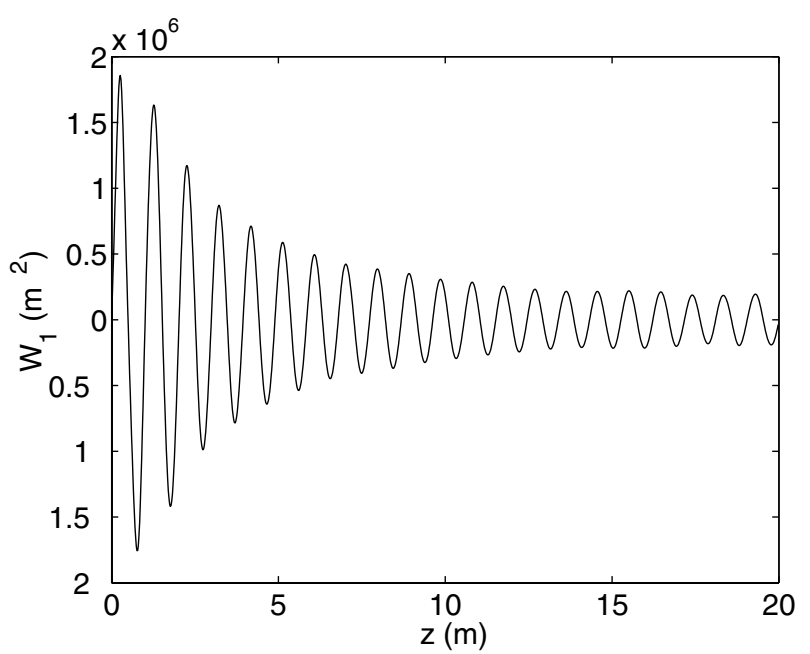

FIG. 9. Wake field caused by electron cloud for J-PARC $50 \mathrm{GeV}$ at injection. 
TABLE III. Wake field and stability for electron cloud instability.

\begin{tabular}{ccccccccc}
\hline \hline \multicolumn{7}{c}{$3 \mathrm{GeV}$} & \multicolumn{2}{c}{ J-PARC } \\
Variable & Inj. & Ext. & Inj. & Ext. & PSR & ISIS & SNS & AGS \\
\hline$Z\left(\omega_{e}\right)_{1, L} / Q(\mathrm{M} \Omega / \mathrm{m})$ & 0.29 & 0.24 & 0.68 & 0.019 & 0.46 & 0.0051 & 0.09 & 0.024 \\
$Z\left(\omega_{e}\right)_{1, H} / Q(\mathrm{M} \Omega / \mathrm{m})$ & 0.61 & 0.83 & 9.7 & 0.96 & 0.90 & 0.0085 & 0.19 & 0.37 \\
$\omega_{e} \ell_{p} / c$ & 133 & 182 & 199 & 276 & 166 & 27 & 272 & 153 \\
$U_{L}$ & 0.07 & 0.23 & 0.11 & 0.02 & 1.6 & 0.007 & 0.30 & 0.004 \\
$U_{H}$ & 0.15 & 0.78 & 1.6 & 1.2 & 3.2 & 0.012 & 0.61 & 0.06 \\
\hline \hline
\end{tabular}

The resonator parameters, obtained by fitting the figure, are as follows:

$$
\begin{aligned}
\frac{c R_{s}}{Q} & =1.9 \times 10^{6} \mathrm{~m}^{-2} \quad\left(1.1 \times 10^{6} \mathrm{~m}^{-2}\right) \\
\omega_{e} & =1.9 \times 10^{9} \mathrm{~s}^{-1} \quad\left(1.2 \times 10^{9} \mathrm{~s}^{-1}\right) \\
Q & =13 .
\end{aligned}
$$

The numbers enclosed in parentheses are the analytical values given by Eqs. (12) and (15). $c R_{S} / Q$ and $\omega_{e}$ are somewhat larger than the analytical ones. $Q=13$ was obtained with the simulation by taking into account the nonlinearities of the beam-cloud interaction. The $Q$ value is conjectured to be further reduced if one considers the longitudinal proton distribution and the modulation of the beta function.

Corresponding effective transverse impedance is given by a Fourier transformation of the wake field.

$$
\begin{aligned}
Z_{1}(\omega) & =\frac{c}{\omega} \frac{R_{S}}{1+i Q\left(\frac{\omega_{e}}{\omega}-\frac{\omega}{\omega_{e}}\right)} \\
& =\frac{\lambda_{e}}{\lambda_{p}} \frac{L}{\sigma_{y}\left(\sigma_{x}+\sigma_{y}\right)} \frac{\omega_{e}}{\omega} \frac{Z_{0}}{4 \pi} \frac{Q}{1+i Q\left(\frac{\omega_{e}}{\omega}-\frac{\omega}{\omega_{e}}\right)},
\end{aligned}
$$

where $Z_{0}$ is the vacuum impedance $377 \Omega$.

We discuss the stability of a beam which experiences the effective impedance. Since the bunch length is very long, $\omega_{e} \ell / c \gg 1$, the coasting beam approximation is used. The stability criterion is given by the dispersion relation as follows [23]:

$$
U \equiv \frac{\sqrt{3} \lambda_{p} r_{p} \beta \omega_{0}}{\gamma \omega_{e} \eta \sigma_{\delta E / E}} \frac{\left|Z_{1}\left(\omega_{e}\right)\right|}{Z_{0}}=\frac{\sqrt{3} \lambda_{p} r_{p} \beta}{\gamma \nu_{s} \omega_{e} \sigma_{z} / c} \frac{\left|Z_{1}\left(\omega_{e}\right)\right|}{Z_{0}}=1,
$$

where $\beta$ is a typical value of the beta function in a ring. For $U>1$, the beam is unstable. $U$ for various rings are calculated using Eq. (15) and the parameters in Tables I and II. We use 5 and $10 \mathrm{~m}$ for $Q$ and $\beta$, respectively. The results on the stability are shown in Table III.

The table includes two values of $U_{H}$ and $U_{L}$, which are the criteria for the peak and the bottom values of the neutralization factor, respectively. The same applies for $Z_{1, H(L)}$.
This result, which shows that the $e^{-} p$ instability is serious for PSR but is not for ISIS, is consistent with experimental results. In J-PARC, $U_{H}$ sometimes exceeds 1.

\section{CONCLUSION}

We discussed the electron cloud buildup and instability in some high intensity proton rings shown in Table I. We studied the electron cloud buildup using a computer simulation. Primary electrons were produced at the chamber surface or at the beam position. We considered electron yields for a proton over a travel distance of $1 \mathrm{~m}, Y_{1, \ell}=$ $4.4 \times 10^{-6} /(\mathrm{m} \cdot p)$, at the chamber surface. The electrons appear due to proton loss, ion hitting, or other mechanisms. The ionization yield was $Y_{1, i}=7.7 \times$ $10^{-9} /(\mathrm{m} \cdot p)\left(2 \times 10^{-7} \mathrm{~Pa}\right)$. The ionization electron can be neglected for a vacuum pressure less than $10^{-5} \mathrm{~Pa}$, if we use the yield $Y_{1, \ell}$ from the chamber surface.

The primary electrons are amplified by the secondary electron emission at the chamber surface. The electrons experience the energy gain due to the beam force, create secondary electrons, and cause multipacting. We calculated the amplification rate for field-free regions. The amplification rates for some proton rings were estimated to be of the order of 5-100 in the present model. The neutralization factors for them are summarized in Table II. We have to note that the rate depends on the secondary yield, beam shape, and chamber geometry. It must be kept in mind that $Y_{1}$ is difficult to estimate in individual machines.

In this calculation, the space charge force between electrons and the elastic scattering (reflection) of electrons [17] is not considered. It may be important, because elastic scattering continues to supply electrons up to the space charge limit. We should not discard the ionization as an electron source [26].

The secondary electrons play an important role for the electron cloud instability in proton rings. Application of a TiN coating to reduce the secondary yield is a very powerful cure for this instability.

The beam stability is estimated by tracking simulations and the coasting beam model using the wake field due to the electron cloud. The estimation is performed 
under the assumption that the whole of the ring was covered by a field-free region. The results are summarized in Table III. The tracking simulation showed results consistent with the coasting beam model. In our calculation, PSR was most severely affected by the instability, while ISIS was safe. J-PARC $3 \mathrm{GeV}$ and SNS are in between PSR and ISIS. For large scale rings, AGS is safe. J-PARC $50 \mathrm{GeV}$ is more affected than AGS. Since the stability criterion $U$ exceeds 1 at the peak cloud density for the $50 \mathrm{GeV}$ ring and is close to 1 for the $3 \mathrm{GeV}$ ring, we have to take care of the instability. We should estimate the cloud density more carefully, including the space charge between electrons, elastic scattering of electrons, effects of lattice magnets, etc.

We investigated the electron cloud buildup in a strong dipole field. The cloud density was suppressed to be about $1 / 4 \sim 1 / 8$ for that of field-free regions in our model. The suppression may depend on the model. The beam electron cloud interaction in a strong dipole field has been discussed for SPS and KEKB [27]. It reported that the dipole field completely suppressed the horizontal instability and slowed down the vertical one. Because of the suppression of electron cloud buildup and of the interaction with the beam, our results may be somewhat pessimistic, but provide a safety margin at the design of accelerators.

\section{ACKNOWLEDGMENTS}

The authors thank the members of the electron-proton instability working group of J-PARC, N. Hayashi, Y. Irie, S. Kato, K. Satoh, and M. Uota, for fruitful discussions. Precious information on PSR and ISIS is given by R. J. Macek and C.R. Prior, respectively. The authors also thank E. Forest and A. Valishev for reading this manuscript.

[1] H. G. Hereward, CERN Report No. 71-15, 1971.

[2] E. Keil and B. Zotter, CERN Report No. CERN-ISR-TH/ 71-58, 1971.

[3] R. J. Macek, A. Browman, D. Fitzgerald, R. C. McCrady, F. E. Merrill, M. A. Plum, T. Spickermann, T.-S. Wang, K. C. Harkay, R. Kustom, R. A. Rosenberg, J. E. Griffin, K. Y. Ng, and D. Wildman, in Proceedings of the Particle Accelerator Conference, Chicago, 2001 (IEEE, Piscataway, NJ, 2001), p. 688.

[4] M. Blaskiewicz, in Workshop on Instabilities of High Intensity Hadron Beams in Rings, edited by T. Roser and S. Y. Zhang, AIP Conf. Proc. No. 496 (AIP, New York, 1999), p. 321.

[5] O. Gröbner, in Proceedings of the 10th International Accelerator Conference, Serpukov, 1977 (Serpukov, Protovino, 1977), Vol. 2, p. 277.

[6] M. A. Furman and M. Pivi, Proceedings of the Particle Accelerator Conference, Chicago, 2001 (Ref. [3]), p. 707.
[7] M. A. Furman and M. Pivi, in Proceedings of the 8th European Particle Accelerator Conference, Paris, France, 2002 (CERN, Geneva, 2002), p. 1547; M. Pivi and M. A. Furman, in Proceedings of the ECLOUDO2 Workshop (CERN Report No. CERN-2002-001, 2002, p. 137.

[8] M. Blaskiewicz, in Proceedings of the 1999 Particle Accelerator Conference, New York (IEEE, Piscataway, NJ, 1999), p. 1611; Proceedings of the 2000 European Particle Accelerator, Vienna (European Physical Society, Geneva, 2000), p. 1110.

[9] V. Danilov, A. Aleksandrov, M. Blaskiewicz, and J. Wei, in Proceedings of the Particle Accelerator Conference, Chicago, 2001 (Ref. [3]), p. 1749.

[10] K. Ohmi and F. Zimmermann, Phys. Rev. Lett. 85, 3821 (2000).

[11] K. Ohmi, F. Zimmermann, and E. Perevedentsev, Phys. Rev. E 65, 016502 (2002).

[12] R. J. Macek (private communication).

[13] Y. Mori (private communication).

[14] Y. Baconnier, CERN Report No. 85-19, 1985, p. 267.

[15] M. A. Furman and G. R. Lambertson, in Proceedings of MBI97 (KEK Report No. 97-17, 1997), p. 170.

[16] T. Toyama, Y. Irie, S. Kato, K. Ohmi, C. Ohmori, K. Satoh, and M. Uota, in Proceedings of the MiniWorkshop on Electron-Cloud Simulations for Proton and Positron Beams, 2002 (CERN Report No. CERN2002-001, 2002).

[17] V. Baglin, I. Collins, B. Henrist, N. Hilleret, and G. Vorlaufer, LHC-Project-Report-472, 2002.

[18] K. Ohmi, Phys. Rev. Lett. 75, 1526 (1995).

[19] L. Wang, H. Fukuma, K. Oide, and S. Kurokawa, Phys. Rev. E (to be published).

[20] M. Blaskiewicz, in Proceedings of 8th ICFA Beam Dynamics Mini-Workshop, Santa Fe, 2000. See http:// w w w. ap s. anl.gov/conferences/icfa/two-stream. html; Proceedings of ECLOUD02, see http://conf-ecloud 02.web.cern.ch/conf-ecloud02/talks/mikeblask.pdf.

[21] T.S. Wang, in Proceedings of 8th ICFA Beam Dynamics Mini-Workshop, Santa Fe, 2000 (Ref. [20]); T. S. Wang, P. J. Channell, and R. J. Macek, in Proceedings of the Particle Accelerator Conference, Chicago, 2001 (Ref. [3]), p. 704.

[22] H. Qin, R. C. Davidson, E. Startsev, and W.W. Lee, in Proceedings of the Particle Accelerator Conference, Chicago, 2001 (Ref. [3]), p. 693.

[23] A.W. Chao, Physics of Collective Beam Instabilities in High Energy Accelerators (Wiley-Interscience Publication, New York, 1993).

[24] K.Y. Ng, in Proceedings of 8th ICFA Beam Dynamics Mini-Workshop, Santa Fe, 2000 (Ref. [20]).

[25] J. Wei et al., in Proceedings of the 8th European Particle Accelerator Conference, Paris, France, 2002 (Ref. [7]), p. 1067.

[26] F. Zimmermann, in Proceedings of Chamonix XI (Cern Report No. CERN-SL-2001-003 DI, 2001).

[27] L. Wang, G. Rumolo, F. Zimmermann, and K. Ohmi, CERN Report No. SL-2001-058 (AP), 2001. 